\title{
Tracking a Moving Object according to the Angle and the Direction via a Smartphones over GPS plus Wi-Fi Coverage with GPRS/3G
}

\author{
Ibrahim Abdallah Hag \\ Eltoum \\ Department of Computer \\ Engineering \\ Laboratory of the Computer \\ System and Telecomm "LIST" \\ FST, Abdelmalek Essaadi \\ University, Tanger, Morocco
}

\author{
Pr. Mohammed \\ Bouhorma \\ Department of Computer \\ Engineering \\ Laboratory of the Computer \\ System and Telecomm "LIST" \\ FST, Abdelmalek Essaadi \\ University, Tanger, Morocco
}

\author{
Tarik Ait Otman \\ Laboratory for Innovative \\ Technology (LTI) \\ The National School of \\ Applied Sciences (ENSAT); \\ Abdelmalek Essaadi \\ University, Tanger, Morocco
}

\begin{abstract}
Smartphones which have a lot of characteristics appear recently and that enable them to compete or replace the computer most of the time specially in dealing which internet and services they require high potentials (processing, memory .. ect) and modern technologies in specific and we must not forget to mention that smart device have other potentials, like dealing with Wi-Fi, GPS, GPRS/3G and the programs which increase the willingness and desire to have them by all. While dealing with GPS in high places, metro or open area which has a thick cover of tress there it face the problem of losing GPS signal or coverage, at the same time a Wi-Fi network can be found available. This guide to look for a mechanism to solve this problem, This paper present a model to trace and determine locations by making use of Wi-Fi which is provided in Smartphones devices and this is to work out the problem mentioned above.
\end{abstract}

\section{Keywords:}

Tracking a Moving Object; GPS plus Wi-Fi; GPRS/3G; Smartphones

\section{INTRODUCTION}

Nowadays GPS is considered a fully functional satellite based positioning system. It is widely used in many fields all over the world. However it has its foibles such as it takes some time to get the first fix; it does not perform well indoors or in urban valleys. GPS need to receivers "see" at least 3 satellites which are relatively well distributed in the sky to calculate its 2D position. Hence in environments where the sky is blocked, positioning becomes difficult; even impossible. In indoor environments, some alternative systems, like active badge cricket [17] etc., have been developed for positioning, but they cannot be used widely due to their inherent problems. Outdoors, mobile phone networks, television signals [14] and pseudolites [12] can be utilized. Since Wi-Fi technologies have been widely utilized, a large number of access points (APs) have been used both indoors and outdoors. Wi-Fi positioning technology has drawn the attention of both researchers and companies [16]. Wi-Fi aims to provide local wireless access to fixed network architectures. Its market is growing rapidly as the flexibility; connectivity, mobility, and low cost of this technology meet the needs of consumers.

It is clear that, Wi-Fi is not designed or used for the purpose of positioning, knowing the location of a user have been used to create location aware application since the early days of pervasive computing. An early example of this is a phone call routing system, which directs the incoming phone calls to the phone nearest to the recipient [20]. Several indoor guide systems for museums and universities have been developed over the years [8]. We have traced and investigated the problem of the losing signal in a Smartphone when GPS system used. In this study the application used to locate an object depending on GPS + Wi-Fi.

The rest of the paper organized as follows: Section 2 overview of the Smartphone; Section 3 the operating system built in it. Sections 4 discuss relevant works. Section 5 included of detailed description of the Features of the Mobile system localization. Section 6 gives an overview of system. Discuss the presented the experimental results in Section 7. Conclude in Section 8.

\section{Smart phones}

It can identify Smartphones as mobile phones built on an operating system like android, with more advanced computing capability and connectivity than a feature phone. Day after day Smartphones are becoming more popular. As mobile sensor platforms are becoming smaller and lower priced while the processing and broadcasting capabilities of these platforms are advancing, the deployment of mobile sensors increases in many different applications. These applications range from environmental monitoring over early warning systems up to military applications.

\section{Android Mobile Development Environment}

Smartphones operates with the basic parts of software system. This operating system which has been designed for these smart devices should have sufficient energy with fewer memory footprint and more development and optimizations, there are numbers of mobile development environments in the market. One of which is Android created by the Open Handset Alliance. Android is an open and comprehensive platform for mobile devices. It is designed to be more open than other mobile operating systems so that developers, wireless operators, and handset manufacturers will be able to make new products faster and at a much lower cost. The result will be a more personalized and more flexible mobile experience to the user. And this why this mobile development 
environment was used in the implementation of the management system.

\section{RELATED WORK}

A wide range of technologies for location tracking have been developed:

The Global Positioning System (GPS) [4] (eg. used in a power saving solution on a mobile phone [3] is yet another system that requires large investment into an infrastructure and expensive administration, but since this has already been implemented and is available for the public throughout the world it seems like a good choice for location tracking, especially when the number of Smartphones embedded with GPS receivers are becoming a commodity. GPS works well in the outdoor environment, but the GPS signals are not powerful enough to be useful inside a building [1], or at places where signals from the satellites are being obstructed. Recent research suggests that in the future this might change as the receivers become more sensitive $[5,1]$.changes in air pressure [9], power lines as signal carriers [11], IR beacons [20], pressure sensors embedded in floors, differences in timeof-flight using both ultra sonic and RF signals in conjunction [21, 19], RFID (eg. using products from [25, 22], ultra wide band RF [24], or using cameras and computer vision to track items or people $[8,18$,$] , to name a few. Although these$ technologies are proven usable they all share the common fact that they are depended on specialized hardware, and might need an infrastructure to interpret the location. This increases the cost of large scale deployment, and might not scale very well. Our project depend on using an already existing infrastructure, users already owned devices, must be highly scalable, easy deployable, and without violating the control over privacy.

Signals from GSM mobile phone antennas have also been utilized and used [10, 17, 13], and unless the signal is very weak (eg. in areas with poor coverage) the signals can travel through buildings. GPS and GSM signals are RF signals with different bandwidths. Utilization of other RF technologies is ZigBee 802.14 [7], Bluetooth [13, 14, 2], FM-antennas [15], antennas broadcasting digital TV signals [6], and Wi-Fi signals 802.11. Research on using Wi-Fi signals for location will be addressed below. Although antennas are widely deployed, special hardware must be embedded into the location tracking device to use this in our project. Only GSM, Bluetooth and Wi-Fi signals are detectable indoor on a device like a mobile phone.

\section{Features of the Mobile system localization}

\subsection{Mobile-centered or infrastructure-centered computation}

There are two ways of operation in each infrastructuresupported positioning system with bidirectional communication. The first one is for the mobile itself to compute its position. In that case, the mobile listens to information transmitted by the infrastructure and computes its position. This information can simply be the Wi-Fi beacons transmitted by access points (APs), in such a case it is simple to add APs to refine the position computation (however we need to beware of the mutual jamming of the APs). The counterpart is the mobile need to embed software which includes an up-to-date list of the APs and their positions. The second possibility is that the mobile may request the infrastructure to compute and send back its position. The advantages of this solution are multiple. First, the mobile only needs a small program to contact the infrastructure and request its position. But the main interest resides in the flexibility provided to the infrastructure for computing. Since the "APs" listening for the mobile requests do not have to transmit themselves, they can be entirely passive and this way avoid polluting the signal environment 3 ; we can then multiply and deploy them without any effect on the wireless network service quality. Infrastructure elements can also easily communicate, coordinate themselves, and we can imagine that the system would be able to adapt itself to environment evolutions.

\subsection{Explicit to implicit positioning requests}

In most cases, the mobile transmits a specific packet, the positioning request; it is an explicit request to the infrastructure. Please note that actually, the only information needed to compute the mobile position is the SS (that the APs get from the mobile), so the infrastructure may make use of any packet transmitted by the mobile; hence, all such packets could be considered as implicit positioning requests: the mobile sends a packet, without explicitly requesting its position, but the infrastructure can make use of it to compute the position. The only missing information in these implicit requests is the time of emission, which could be replaced by a hash sum.

\section{System overview}

The proposed tracking system in this paper is designed to track and monitor Smartphones, status that is used by certain party for particular purposes; this system is an integration of several modern embedded and communication technologies. To provide location and time information anywhere in the world, GPS + Wi-Fi "built in the Smartphone" is commonly used as a space-based global navigation satellite system. The location information provided can be visualized using Google Earth. The path of this application can monitor the location graphically on Google Earth; it also can view other relevant information of each Smartphone in the fleet by using Real Time online method. In case they is no network, the Smartphone save the track points and when it rich network, will send the path of the object.

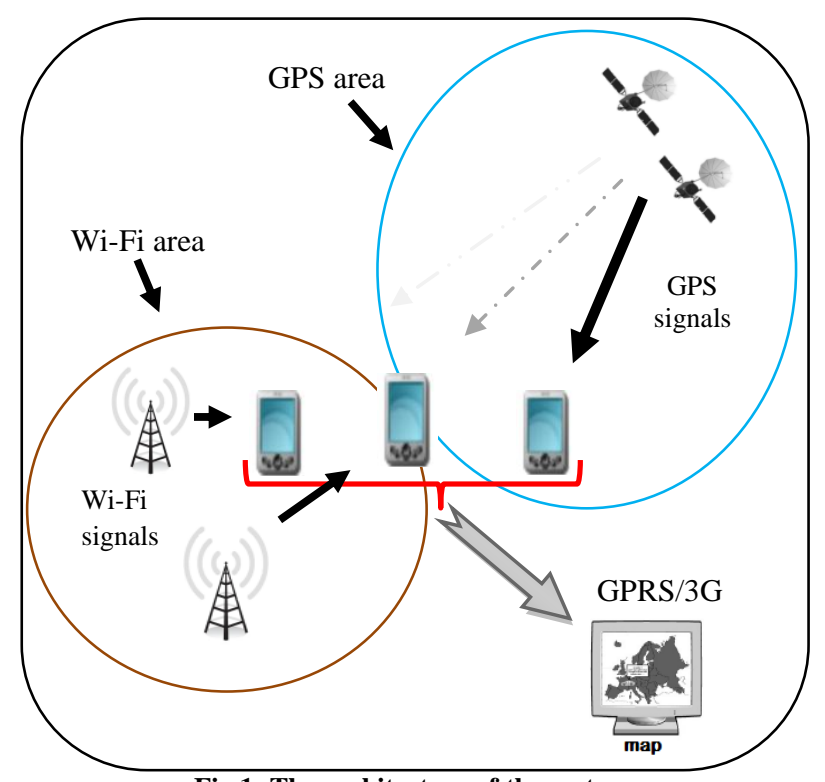

Fig 1: The architecture of the system. 


\subsection{The mathematic model}

The below equation works in finding the angle of the object depending on the obtained points $\mathrm{P}_{\mathrm{n}-1} \& \mathrm{P}_{\mathrm{n}}$ :

$$
\begin{aligned}
& M=m=\tan \varnothing=\frac{y_{n-1}-y_{n}}{x_{n-1}-x_{n}} \ldots \ldots \ldots \ldots 1 \\
& \rightarrow \varnothing=\tan ^{-1} m \ldots \ldots \ldots \ldots \ldots \ldots \ldots 1
\end{aligned}
$$

To find the distance between any two points the following form can use:

$$
\Delta \mathrm{d}=\sqrt{\left(x_{n}-x_{n-1}\right)^{2}+\left(y_{n}-y_{n-1}\right)^{2}}
$$

The time which the object needs to move from Pn-1 until it reaches Pn will be use:

$$
\Delta \mathrm{t}=\mathrm{TP}_{\mathrm{n}}-\mathrm{TP}_{\mathrm{n}-1} \ldots \ldots . .3
$$

$\Delta \mathrm{d} \& \Delta \mathrm{t}$ found in equation $2 \& 3$ are used to find the speed of the moving object and calculate the velocity as follows:

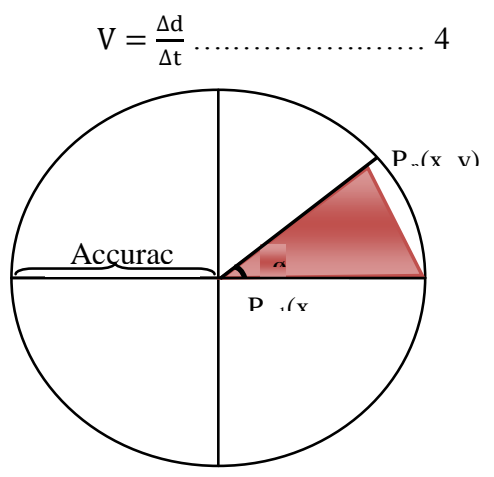

Fig 2: the search space of the object.

\subsection{Positioning Algorithm}

* To determine location depending on the GPS, its compares all the available decisions by GPS "All Satellites" in addition to all other decisions obtained by smart device through available technology provided on it and chooses the most accurate one. In case of weakness or losing signal from GPS measuring will be obtained through the Wi-Fi available, to measuring the location.

* To increasing the accuracy in the $\mathrm{Wi}-\mathrm{Fi}$, using the angle \& direction of the movement, equation (2) with applied to obtain slope of the straight object which links the last measuring of GPS "after losing or weakness of signal" and the first point measuring it with Wi-Fi.

* The time that the object takes moving from the point $\mathrm{P}_{\mathrm{n}-1}$ to $\mathrm{P}_{\mathrm{n}}$ is $\Delta \mathrm{t}$ and to obtain the distance $\mathrm{D}$ between two points, the equation (2) was used.

$* \Delta \mathrm{d} \& \Delta \mathrm{t}$ utilize in equation (3) to calculate the speed of the object.

\subsubsection{Determining the search part}

* To determine the place and direction of the traced target movement we compare the value of the point $P_{n-1}, P_{n}$ as follows:

- If the values $x$, $y$ of $P_{n}$ greater than $P_{n-1}$ that means it situated in the first part $\rightarrow(+,+)$.

- If the values $\mathrm{x}, \mathrm{y}$ of $\mathrm{P}_{\mathrm{n}-1}$ greater than $\mathrm{P}_{\mathrm{n}}$ that means it situated in the third part $\rightarrow(-,-)$.
- if the values are different,

- Where $y$ of $P_{n}$ greater than y of $P_{n-1}$ that means it situated in the second part $\rightarrow(-,+)$.

$\circ \quad$ Where $x$ of $P_{n}$ greater than $x$ of $P_{n-1}$ that means it situated in the fourth part $\rightarrow(+,-)$.

\subsubsection{Reducing the search-space}

To reduce the space, waiting up the object reach $\mathrm{P}_{\mathrm{n}+1}$, then calculate the $\emptyset_{2}$. Before that using $\emptyset_{1} \& \emptyset_{2}$ to get $\Delta \varnothing$ :

$$
\Delta \emptyset=1 \emptyset_{2}-\emptyset_{1} 1 \ldots \ldots . .5
$$

In the most cases $2 \Delta \varnothing<=\varnothing 1, \varnothing_{2}$, unless if the object is moving very fast ( $\Delta \varnothing \propto \mathrm{V})$ and the range of search well be: $\emptyset_{2} \pm \Delta \emptyset$ or $2 \Delta \emptyset$, when $\Delta \emptyset=0 \ni \mathrm{P}_{\mathrm{n}}=\mathrm{P}_{\mathrm{n}+1}$.

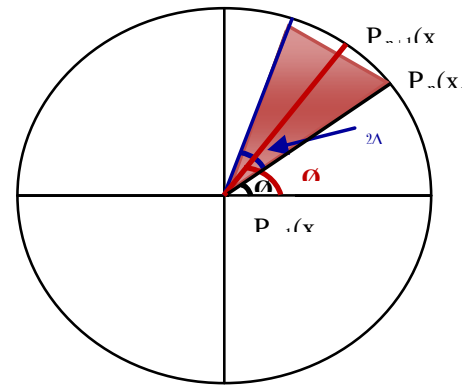

Fig 3: search space according to $\Delta \varnothing$.

* After getting the location from Wi-Fi and determining the position then recheck the signal of GPS if found use the GPS to get the next position, otherwise keep on using Wi-Fi for the positioning, after every positioning look for GPS single if found go back to GPS mode.

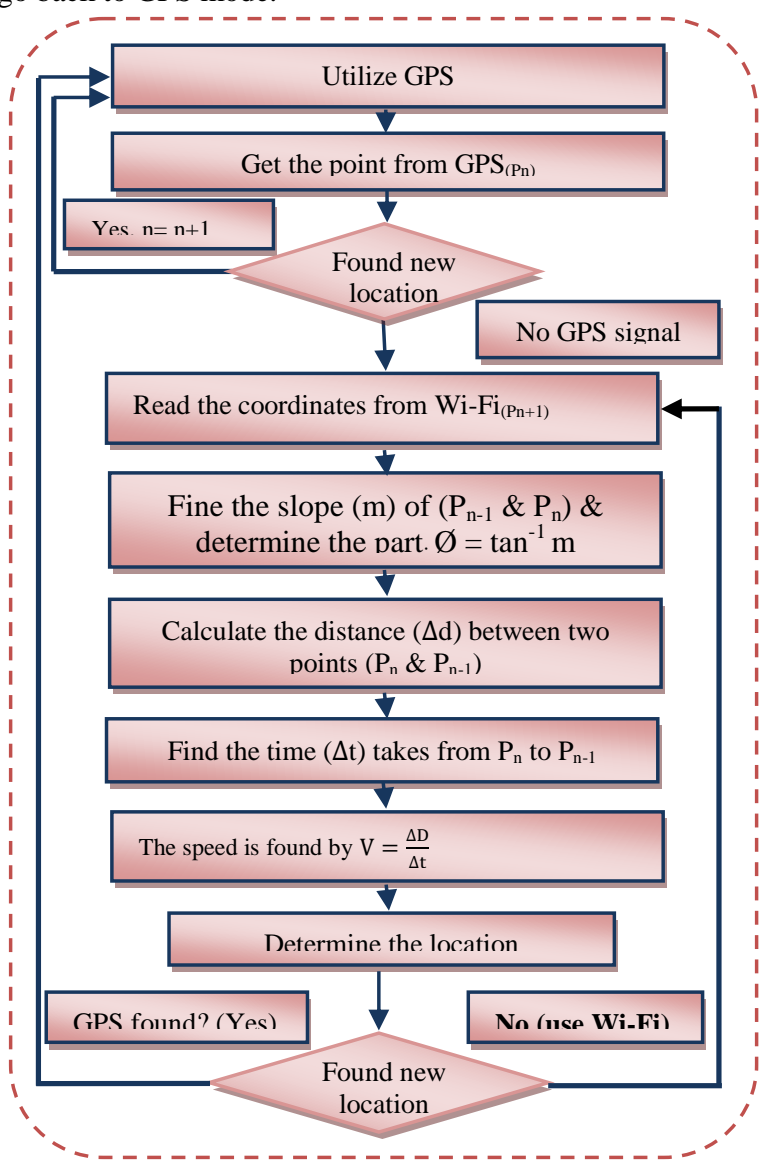

Fig 4: positioning algorithm 


\section{RESULT}

This paper developed application according to GPS plus WiFi to determine the location, instead of searching in circle in which the object is supposed to be in its centre (the point obtained by GPS) the circle radius equal the accuracy. The values of the moving angle restrict the research into a specific place in a definite part of the circle up to quarter of the circle. Based on the value of $\Delta \varnothing$ can predict the acceleration of body movement and regularity and through $\Delta \varnothing$ the search space is reduced up to the lowest value of possible. The application used the object speeds to increase the accuracy of tracking.

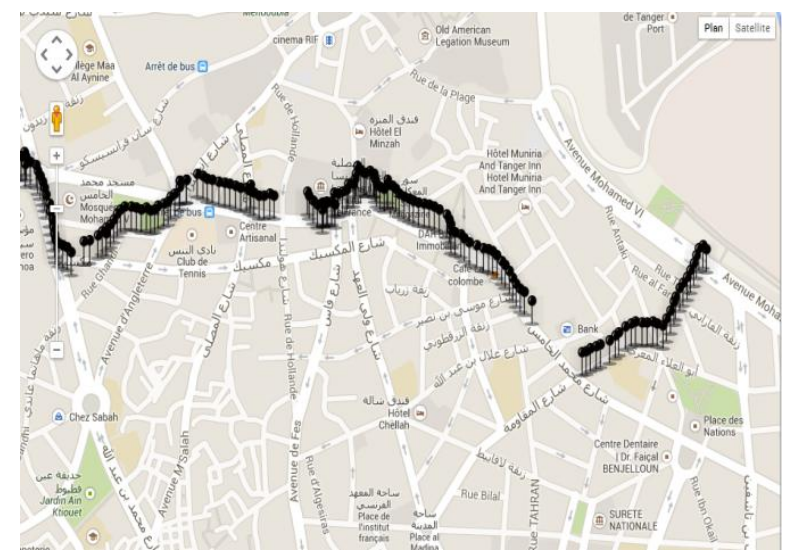

Fig 5: When GPS Signals loss.

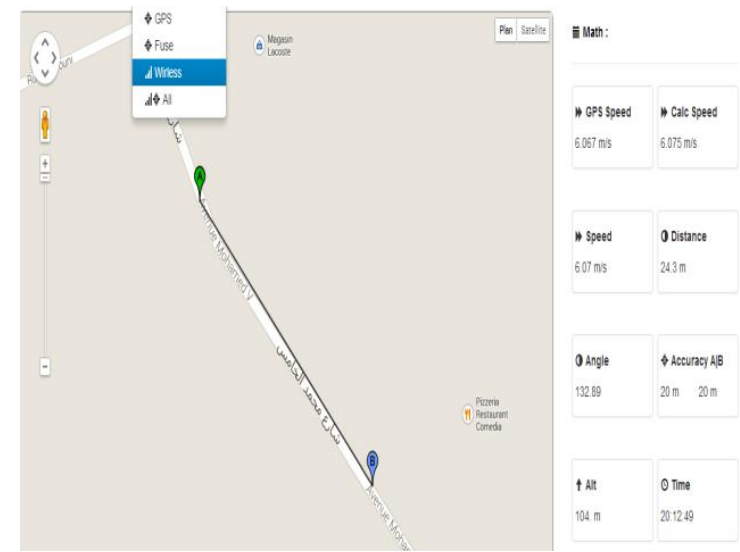

Fig 6: When Using Wi-Fi Signals.

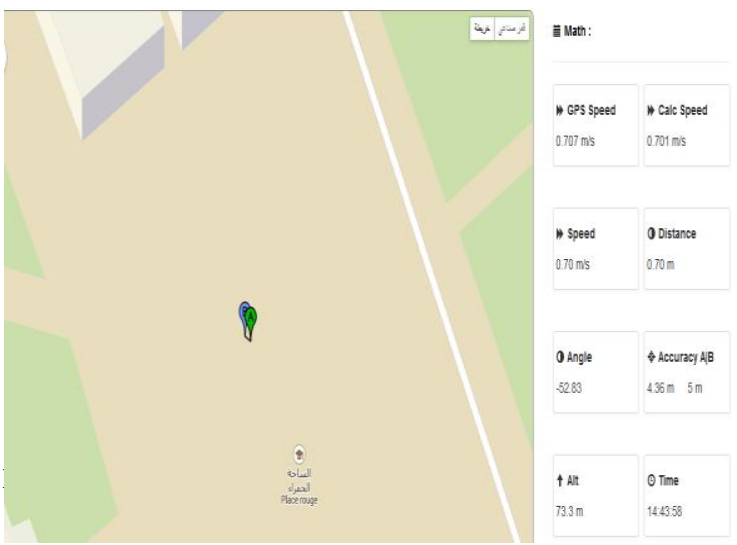

This study presented a reasonable view to the possibility of determining location using technologies in smart devices such as GPS, Wi-Fi and all mechanisms used to determine the position in these devices. It is compatible only with Android
Application; it is a real-time location tracking integration with GPS plus Wi-Fi and GPRS/3G techniques. The following is found: the application makes the determination of location more accurate, reduce the effort; the speed used to reach more accrues that reached a range of 1 meter/sec, gaining a bigger $\&$ better area of coverage, which can be used in GPS or WiFi.

\section{REFERENCE}

[1] Ibrahim Abdallah Hag Eltoum, M. Bouhorma: Velocity based Tracking and Localization System Using Smartphones with GPS and GPRS/3G, IJCA, V 76, August 2013.

[2] B. S. A/S. Cph airport expands bliptrack solution. http://www.blipsystems.com/Default.aspx ?ID=1267\&Ac tion $=1 \&$ NewsId $=205 \& M=$ NewsV2\&PID $=5594$, June 2010 .

[3] I. Constandache, R. R. Choudhury, and I. Rhee. Towards mobile phone localization without war-driving. In INFOCOM, pages 2321-2329. IEEE, 2010.

[4] P. Enge and P. Misra. Special issue on gps: The global positioning system. In Proceedings of the IEEE, pages 3 172, Jan. 1999

[5] M. B. Kjærgaard, H. Blunck, T. Godsk, T. Toftkjær, D. L. Christensen, and K. Grønbæk. Indoor positioning using GPS revisited. In Proceedings of the Eight International Conference on Pervasive Computing, 2010.

[6] B. Rong, B. Liu, Y. Wu, G. Gagnon, L. Gui, and W. Zhang. Mobile location finding using ATSC mobile/handheld digital TV RF watermark signals. In VTC Fall, pages 1-5, 2010.

[7] M.-H. Hung, S.-S. Lin, J.-Y. Cheng, and W.-L. Chien. A zigbee indoor positioning scheme using signal-index-pair data preprocess method to enhance precision. In ICRA, pages 548-553. IEEE, 2010.

[8] J. Krumm. Ubiquitous Computing Fundamentals. Chapman \& Hall/CRC, 1st edition, 2009.

[9] S. N. Patel, M. S. Reynolds, and G. D. Abowd. Detecting human movement by differential air ressure sensing in HVAC system ductwork: An exploration in infrastructure mediated sensing. In J. ndulska, D. J. Patterson, T. Rodden, and M. Ott, editors, Preceeding of the International Conference on Pervasive Computing (Pervasive), volume 5013, pages 1-18. Springer, 2008.

[10] A. Varshavsky, E. de Lara, J. Hightower, A. LaMarca, and V. Otsason. GSM indoor localization. Pervasive and Mobile Computing, 3(6):698-720, 2007.

[11] S. N. Patel, K. N. Truong, and G. D. Abowd. Powerline positioning: A practical sub-room-level indoor location system for domestic use. In P. Dourish and A. Friday, editors, Ubicomp, volume 4206 of Lecture Notes in Computer Science, pages 441-458. Springer, 2006.

[12] Barnes J.; Rizos C.; Kanli M.; Pahwa A. (2006): A positioning technology for classically difficult GNSS environments from Locata, IEEE/ION PLANS, San Diego, California, 25-27 April, 715- 721.

[13] A. LaMarca, Y. Chawathe, S. Consolvo, J. Hightower, I. Smith, J. Scott, T. Sohn, J. Howard, J. Hughes, F. Potter, J. Tabert, P. Powledge, G. Borriello, and B. Schilit. Place lab: Device positioning using radio beacons in the wild. 
In Pervasive 2005: Third International Conference on Pervasive Computing, pages 116-133. Springer-Verlag, 2005.

[14] Eggert R.J.; Raquet J.F. (2004): Evaluating the navigation potential of the NTSC analog television broadcast signal, ION GNSS $17^{\text {th }}$ International Technical Meeting of the Satellite Division, Long Beach, California, USA, 21-24 September, pp. 2436-2446.

[15] J. Krumm, G. Cermak, and E. Horvitz. RightSPOT: A novel sense of location for a smart personal object. In A. K. Dey, A. Schmidt, and J. F. McCarthy, editors, Ubicomp, volume 2864 of Lecture Notes in Computer Science, pages 36-43. Springer, 2003.

[16] Ladd A.M.; Bekris K.E.; Rudys A.; Marceau G.; Kavraki L.E.; Dan, S. (2002): Robotics-based location sensing using wireless Ethernet, Eighth ACM Int. Conf. on Mobile Computing \& Networking (MOBICOM), Atlanta, Georgia, USA, 23-28 September, pp. 227-238.

[17] Priyantha N.B.; Chakraborty A.; Balakrishnan H.(2000): The cricket location-support system, Proc.6th ACM International Conference on MobileComputing and Networking, Boston, USA, 06-11August, pp.32-43.
[18] J. Krumm, S. Harris, B. Meyers, B. Brumitt, M. Hale, and S. Shafer. Multi-camera multi-person tracking for easyliving. In Proceedings of the Third IEEE International Workshop on Visual Surveillance (VS'2000), VS '00, pages 3-, Washington, DC, USA, 2000. IEEE Computer Society.

[19] N. B. Priyantha, A. Chakraborty, and H. Balakrishnan. The cricket location-support system. In MOBICOM, pages 32-43, 2000.

[20] R. Want, A. Hopper, V. Falcao, and J. Gibbons. The active badge location system. ACM ransactions on Information Systems, 10(1):91-102, Jan. 1992.

[21] A. Ward and A. Jones. A new location technique for the active office. IEEE Personal Communications, 4(5):4247, Nov. 261997.

[22] Alien technology. http://www.alientechnology.com/.

[23] Ubisense. http://www.ubisense.net/.

[24] Wavetrend. http://www.wavetrend.net/. 